technique to detect nucleoprotein (NP) antigens and RT-PCR were positive for pandemic H1N1 influenza. The virus antigen was also detected by flow cytometry using monoclonal antibody specific for NP labeled with fluorescein isothiocyanate (FITC). Interestingly, most of positive cells were CD14+ cells and the amounts of positive cells were $10 \%$ of total marrow cells. His immunological profiles were normal.

Conclusion This infant demonstrated the evidence of disseminated pandemic H1N1 influenza prior having pneumonic symptoms. Infant is more susceptible to have disseminated manifestation and can present initially as clinical sepsis.

\section{ASPERGILLUS NIGER: AN UNUSUAL CAUSE OF INVASIVE PULMONARY ASPERGILLOSIS}

doi:10.1136/archdischild-2012-302724.0522

S Hammami, S Haddad, K Lajmi, N Bel Hadj Brahim, L Ghédira, C Ben Meriem, MN Guediche. Paediatric Department, Faculty of Medicine - Monastir University, Monastir, Tunisia

Objective Report two cases of chronic granulomatous disease presenting with uncommon cause of invasive pulmonary aspergillosis (IPA).

Methods We report two cases of IPA in two 6-year-old twin girls with a family history of primary immunodeficiency and a personal medical history of recurrent bronchopneumopathy referred to our Pediatric Department in July 2011 for investigations regarding persistent pneumonia and biological inflammatory syndrome. IPA was confirmed by isolating Aspergillus niger from bronchoalveolar lavage and radiology results. Amphotericin B therapy was initiated intravenously for 1 month relayed then by oral Voriconazole for two months. Clinico-biological evolution was favourable. Followup computed tomography showed full regression of the pulmonary infiltrates and the thoracic wall mass after 3 months of antifungal therapy. Investigation for immune deficiency revealed chronic granulomatous disease. Our two patients started then on prophylactic antibiotics with co-trimoxazole. We are currently considering HLA identical bone marrow transplantation for them in a near future.

Conclusion Although is a well recognized clinical entity, invasive disease caused by aspergillus niger is less common when compared to aspergillus fumigatus and other Aspergillus species. These two case reports demonstrate the potentially aggressive nature of aspergillus niger and highlight the importance of looking for an immune deficiency particularly in the case of uncommon infection such as aspergillosis in early childhood.

\section{A CASE REPORT; BRUCELLOSIS TRIGGERING HEMOLYTIC ANEMIA IN GLUCOSE-6-PHOSPHATE DEHYDROGENASE DEFICIENCY}

doi:10.1136/archdischild-2012-302724.0523

${ }^{1} \mathrm{M}$ Özkale, ${ }^{1} Y$ Özkale, ${ }^{2} \mathrm{~A}$ Erbay, ${ }^{1} \mathrm{U}$ Paltacı, ${ }^{3} \mathrm{~S}$ Asilsoy. ${ }^{1}$ Pediatric; ${ }^{2}$ Pediatric Oncology; ${ }^{3}$ Pediatric Allergy, Baskent University, Adana, Turkey

Background and Aims Glucose-6-phosphate dehydrogenase (G6PD) deficiency is the most common red blood cell enzyme deficiency in worldwide. It may lead to acute hemolytic anemia triggered by infection, drugs or broad beans (favism). To the best of our knowledge, this is the second reported case of a Turkish patient with brucellosis triggering hemolytic anemia in glucose-6-phosphate dehydrogenase deficiency.

Case A 5-year-old male was admitted our hospital with malaise, fever, abdominal pain, vomiting, jaundice, and darkening of urinary color of one week duration. There were no intake drug, infectious diseases or favism in patient's history. On physical examination conjunctivae were pale, sclerae were subicteric and the other system examinations were normal. Laboratory examinations revealed leukocyte count of $22800 / \mathrm{mm}^{3}$, hemoglobin of $5.7 \mathrm{~g} / \mathrm{dl}$, platelets of $596000 / \mathrm{mm}^{3}$, total bilirubin:4.3, indirect bilirubin:3.9 mg/dl, lactate dehydrogenase:1440U/1, AST:190IU/L, ALT:181IU/L. The corrected reticulocyte count was $9.3 \%$ and serum haptoglobin level was low $(<10)$. There was hemoglobinuria in the urinary examination. Direct and indirect Coombs tests were negative. The value of G6PD was low $(3.8 \mathrm{IU} / \mathrm{g}$ ) where osmotic fragility was normal. Serum standard tube agglutination (Wright) test for Brucella sp was positive at a titer of $1 / 640$. The patient was treated by rifampicin and gentamicin.

Conclusion We presented this case report to show that acute brucellosis might trigger an acute hemolytic attack in a patient with underlying G6PD deficiency. This is important especially in countries such as Turkey, where brucellosis is endemic and there is a high frequency of G6PD deficiency in the population.

\section{TWO PEDIATRIC CASES OF CEREBRAL VENOUS SINUS THROMBOSIS WITH DIFFERENT PRESENTATIONS}

doi:10.1136/archdischild-2012-302724.0524

'B Kiliçaslan, ${ }^{2} \mathrm{E}$ Erol, ${ }^{3} \mathrm{~N}$ Yazıcı, ${ }^{4} \mathrm{O}$ Alkan. ${ }^{1}$ Department of Pediatrics; ${ }^{2}$ Department of Pediatrics, Division of Child Neurology; ${ }^{3}$ Department of Pediatrics, Division of Child Hematology and Oncology; ' Department of Radiology, Baskent University Hospital, Adana, Turkey

Background and Aims Cerebral Venous Sinus Thrombosis (CVST) is a serious and rare disorder in pediatric patients.

Case reports: Case 1: An 8-year-old girl admitted with left middle ear infection, bilateral sixth cranial nerve palsy and papilledema. The Magnetic Resonance Imaging (MRI) venography showed occlusion of superior and inferior sagittal sinus totally and left sigmoid sinüs parsial.Pansinüsitis, left otitis media and mastoiditis were also determined. Evaluation for thrombophilia and tests for specific predisposing conditions were normal. The patient was treated by third generation cephalosporin, clindamycin, acetazolamide and low-molecular weight heparin after the initial diagnosis.

Case 2: A 12-year-old boy presented with aphasia and lethargy after a week of dental infection. Brain MRI and MRI venography demonstrated thalamic enfarct, thrombosis of deep cortical veins, left transverse and sigmoid sinuses.

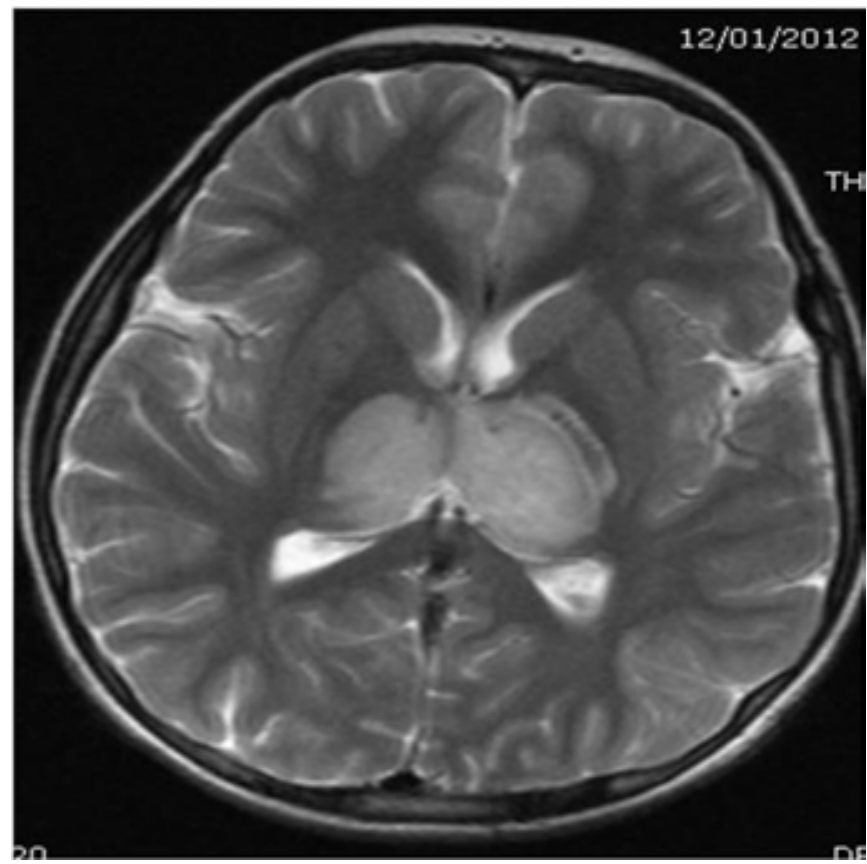

Abstract 524 Figure 1 


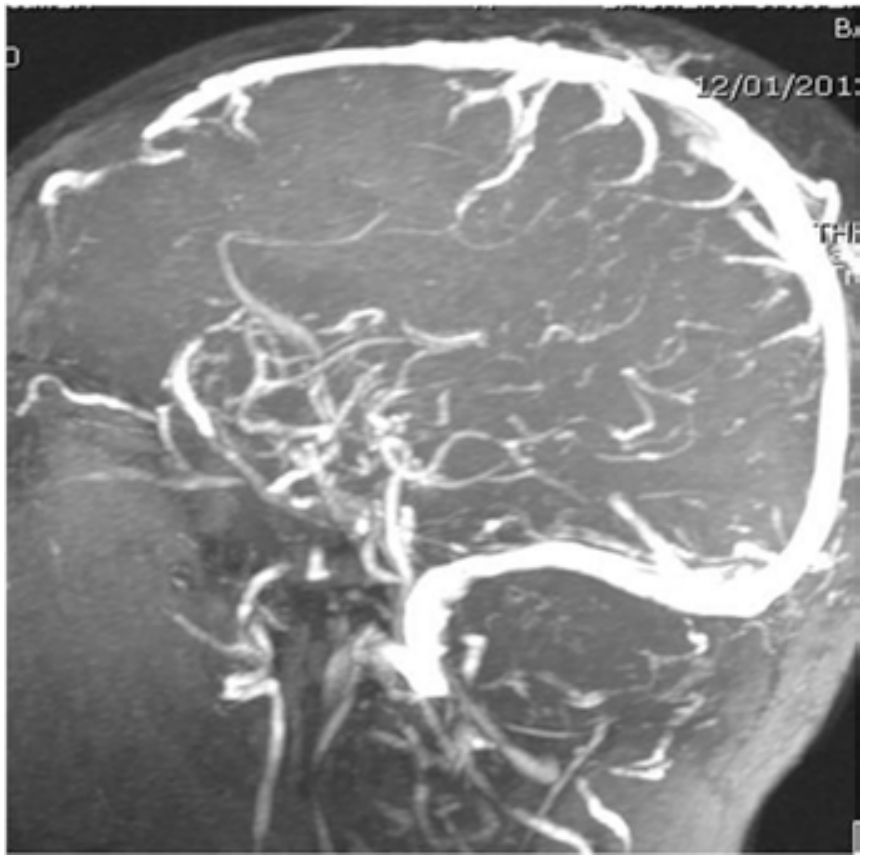

Abstract 524 Figure 2a

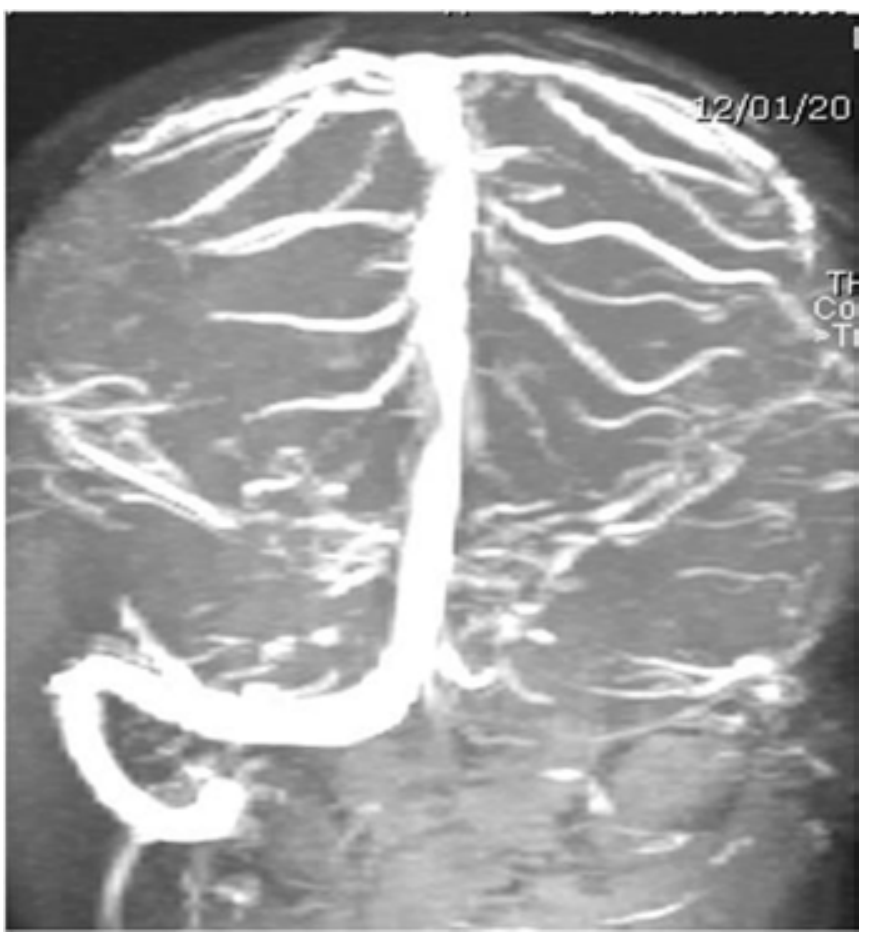

Abstract 524 Figure 2b

Evaluation for thrombophilia and tests for specific predisposing conditions were normal. The patient was treated by third generation cephalosporin, clindamycin and low-molecular weight heparin. Two months after the initial diagnosis, the evidence of a small focal thalamic hemorrhage was noted on MRI. Recanalization of venous occlusions were detected on MRI venography.Low-molecular weight heparin therapy was changed with oral acetylsalicylic acid.

Conclusion Since CSVT is an underrecognized cause of intracranial pathologic abnormality in the pediatric population, we wish to draw the attention of pediatricians to the importance of a high level of suspicion for this diagnosis and the preferred diagnostic method which is MRI with venography.

\section{PAEDIATRIC MOYAMOYA SYNDROME ASSOCIATED WITH IRON DEFICIENCY ANAEMIA: A CASE REPORT AND REVIEW OF THE LITERATURE}

doi:10.1136/archdischild-2012-302724.0525

K McElroy, A Kennedy, L Houliston, A Judkins, G Chaney, P Richmond. Department of Paediatric and Adolescent Medicine, Princess Margaret Hospital for Children, Perth, WA, Australia

Introduction Moyamoya syndrome is a rare, progressive disorder characterised by stenosis of the vasculature of the Circle of Willis with formation of a tortuous collateral system. The aetiology may be idiopathic, or as a consequence of a number of associated diseases. No previous cases secondary to iron-deficiency anaemia have been reported.

Aim To report a case of Moyamoya syndrome secondary to irondeficiency anaemia, and review the literature with regard to the haematological associations of Moyamoya syndrome.

Method The relevant case notes were reviewed. Key databases were searched for studies including the target phrases "Moyamoya syndrome" and "anaemia".

Results Case history: A 3 year old girl presents with a 12 hour history of right upper limb weakness and slurred speech. A background of pallor, daily vomiting and abdominal pain is elicited. Reduced power in the right upper and lower limbs and a right-sided upgoing plantar are noted on examination. Investigations reveal a haemoglobin of $1.9 \mathrm{~g} / \mathrm{dL}$, with an iron-deficiency pattern. A faecal occult blood test is positive. Magnetic Resonance (MR) imaging shows focal middle cerebral artery territory ischaemia. MR Angiography shows Moyamoya vessels. Extensive investigations for associated pathologies are normal.

Literature review: Sickle cell disease is the most common haematological cause of the Moyamoya syndrome, whilst thalassemia, Fanconi's anaemia and paroxysmal nocturnal haemoglobinuria are also reported. The likely pathogenesis in these instances is intimal proliferation in response to impaired arterial flow.

Conclusion We propose that severe iron-deficiency anaemia may result in Moyamoya syndrome through the mechanism of disrupted intracerebral arterial flow.

\section{PROTOCOLS FOR THE TREATMENT OF HODGKIN LYMPHOMA - CASE REPORT}

doi:10.1136/archdischild-2012-302724.0526

Al Krasniqi, V Grajqevci-Uka, R Maqastena-Maxhuni, B Abrashi, F Selimi, E Bajrami, E Islamaj, S Nushi. Hemato-Oncology Department, UCCK, Pediatric Clinic, Prishtina, Kosovo

Introduction Hodgkin lymphoma is malignant disease of cells in the lymphatic system that is characterized by proliferation of Reed Sternberg cells. Symptoms include the painless swelling of lymph nodes, spleen, or other immune tissue. Other symptoms include fever, weight loss, fatigue, or night sweats. Also called Hodgkin disease.

Objective Was the presentation a case with Hodgkin lymphoma, treated in Pediatric Clinic in Prishtina at the Hemato-Oncology Unit.

Methods Patient, male child, 9 years old, came in the clinic because of painless enlargement of lymph nodes on the left side of the neck. Other examinations, according to systems have been in normal range. Diagnosis is made based on anamnesis, clinical examination, laboratory, ultrasound, CT, histopathology with immunochemistry, and bone marrow aspiration. The surgery has been made and according to path-histological and immunochemistry analyses the 